\title{
Component Reuse in Iterative Solvers for the Solution of Fuzzy Partial Differential Equations
}

\author{
Samuel Corveleyn and Stefan Vandewalle
}

Department of Computer Science

Katholieke Universiteit Leuven

September 21, 2009 
- PDEs of elliptic type with fuzzy parameters.

- Our goal is to determine fuzzy characteristics of the PDE solution.

- Problem can be rewritten as large set of related global optimization problems.

- We consider component reuse in function and gradient evaluations to accelerate optimization. 


\section{Outline}

(1) Introduction

(2) Background

- Fuzzy Numbers

- Operations on Fuzzy Numbers

- Optimization with $\mathrm{G} \alpha \mathrm{D}$

- PDE with fuzzy parameters

(3) Component Reuse

- Problem Formulation

- Reuse in matrix assembly phase

- Reuse of earlier solution as initial guess

- Reuse intergrid operators in algebraic multigrid

(4) Numerical Results 


\section{Fuzzy Numbers}

\section{What are they for?}

Increasingly popular method to model uncertainty in engineering applications. Stochastic methods are criticized in case of little knowledge on distribution function. Fuzzy numbers are useful for early design, worst-case analysis (e.g. for structures) and sensitivity analysis.

\section{What are they?}

Special kind of fuzzy set. Fuzzy number $\widetilde{x}$ is represented by membership function $\mu_{\tilde{x}}: \Omega \rightarrow[0,1]$, where the value $\mu_{\tilde{x}}(x)$ represents the degree of membership of $x$. A classical set either contains an element or not, which means its membership function is a function $\mu_{\tilde{x}}: \Omega \rightarrow\{0,1\}$. 


\section{Operations on Fuzzy Numbers}

- The fuzzy extension $\tilde{y}=f\left(\widetilde{x_{1}}, \widetilde{x_{2}}, \ldots, \widetilde{x_{n}}\right)$ of a function $y=f\left(x_{1}, x_{2}, \ldots, x_{n}\right)$ is given by Zadeh's extension principle:

$$
\mu_{\widetilde{y}}(y)=\sup _{y=f\left(x_{1}, x_{2}, \ldots, x_{n}\right)} \min \left\{\mu_{\widetilde{x_{1}}}\left(x_{1}\right), \mu_{\widetilde{x_{2}}}\left(x_{2}\right), \ldots, \mu_{\widetilde{x_{n}}}\left(x_{n}\right)\right\}
$$

- Using the $\alpha$-cut approach, this can be reformulated:

$$
[\widetilde{y}]_{\alpha}=f\left(\left[\widetilde{x_{1}}\right]_{\alpha},\left[\widetilde{x_{2}}\right]_{\alpha}, \ldots,\left[\widetilde{x_{n}}\right]_{\alpha}\right):=\left\{y: y=f(\mathbf{x}), \mathbf{x} \in[\widetilde{\mathbf{x}}]_{\alpha}\right\}
$$

with $[\widetilde{y}]_{\alpha}=\left\{y: \mu_{\widetilde{y}}(y) \geqslant \alpha\right\}$. This is Interval Analysis. 


\section{Example}

$\cos (\widetilde{x})$, where $\widetilde{x}=\operatorname{triag}(-1,1,2)$

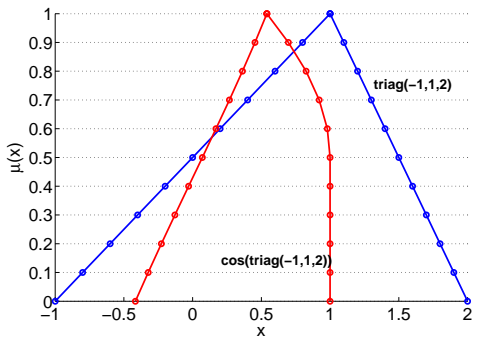

\section{Optimization}

The $\alpha$-cut approach is equivalent to max- and minimization on the search space $[\widetilde{\mathbf{x}}]_{\alpha}$, for every $\alpha$-cut we take. For $f$ continuous:

$$
[\tilde{y}]_{\alpha}=[f(\widetilde{\mathbf{x}})]_{\alpha}=\left[\min _{\mathbf{x}}(f(\mathbf{x})), \max _{\mathbf{x}}(f(\mathbf{x}))\right] \text { s.t. } \mathbf{x} \in[\mathbf{x}]_{\alpha}
$$




\section{Optimization with $\mathrm{G} \alpha \mathrm{D}$}

A dedicated global optimization algorithm:

\section{Gradual $\alpha$-level Decreasing ( $\mathrm{G} \alpha \mathrm{D}$ ) Algorithm}

Optimization on growing hyperrectangular search space from $\alpha$-level 1 to $\alpha$-level 0 .

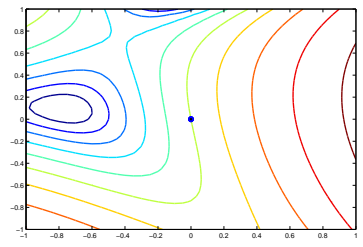

$$
\begin{aligned}
y= & \log \left(x_{1}^{3}+3.2 x_{1}^{2}+3.08 x_{1}+x_{1} x_{2}\right. \\
& \left.+0.4 x_{2}-3 x_{1} x_{2}^{2}-0.2 x_{2}^{2}-x_{2}^{3}+1\right) \\
\widetilde{x}_{1}= & \operatorname{triag}(-1,0,1) \\
\widetilde{x}_{2}= & \operatorname{triag}(-1,0,1)
\end{aligned}
$$

$$
\alpha=1
$$




\section{Optimization with $\mathrm{G} \alpha \mathrm{D}$}

A dedicated global optimization algorithm:

\section{Gradual $\alpha$-level Decreasing ( $\mathrm{G} \alpha \mathrm{D}$ ) Algorithm}

Optimization on growing hyperrectangular search space from $\alpha$-level 1 to $\alpha$-level 0 .

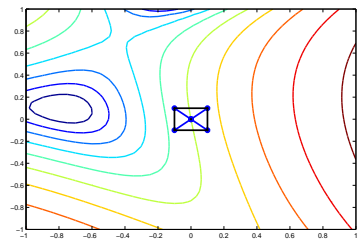

$$
\begin{aligned}
y= & \log \left(x_{1}^{3}+3.2 x_{1}^{2}+3.08 x_{1}+x_{1} x_{2}\right. \\
& \left.+0.4 x_{2}-3 x_{1} x_{2}^{2}-0.2 x_{2}^{2}-x_{2}^{3}+1\right) \\
\widetilde{x}_{1}= & \operatorname{triag}(-1,0,1) \\
\widetilde{x}_{2}= & \operatorname{triag}(-1,0,1)
\end{aligned}
$$

$$
\alpha=0.9
$$




\section{Optimization with $\mathrm{G} \alpha \mathrm{D}$}

A dedicated global optimization algorithm:

\section{Gradual $\alpha$-level Decreasing ( $\mathrm{G} \alpha \mathrm{D}$ ) Algorithm}

Optimization on growing hyperrectangular search space from $\alpha$-level 1 to $\alpha$-level 0 .

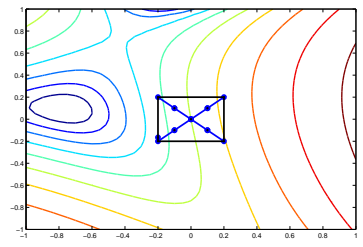

$$
\begin{aligned}
y= & \log \left(x_{1}^{3}+3.2 x_{1}^{2}+3.08 x_{1}+x_{1} x_{2}\right. \\
& \left.+0.4 x_{2}-3 x_{1} x_{2}^{2}-0.2 x_{2}^{2}-x_{2}^{3}+1\right) \\
\widetilde{x}_{1}= & \operatorname{triag}(-1,0,1) \\
\widetilde{x}_{2}= & \operatorname{triag}(-1,0,1)
\end{aligned}
$$

$$
\alpha=0.8
$$




\section{Optimization with $\mathrm{G} \alpha \mathrm{D}$}

A dedicated global optimization algorithm:

\section{Gradual $\alpha$-level Decreasing ( $\mathrm{G} \alpha \mathrm{D}$ ) Algorithm}

Optimization on growing hyperrectangular search space from $\alpha$-level 1 to $\alpha$-level 0 .

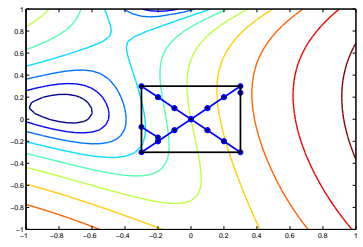

$$
\begin{aligned}
y= & \log \left(x_{1}^{3}+3.2 x_{1}^{2}+3.08 x_{1}+x_{1} x_{2}\right. \\
& \left.+0.4 x_{2}-3 x_{1} x_{2}^{2}-0.2 x_{2}^{2}-x_{2}^{3}+1\right) \\
\widetilde{x}_{1}= & \operatorname{triag}(-1,0,1) \\
\widetilde{x}_{2}= & \operatorname{triag}(-1,0,1)
\end{aligned}
$$

$$
\alpha=0.7
$$




\section{Optimization with $\mathrm{G} \alpha \mathrm{D}$}

A dedicated global optimization algorithm:

\section{Gradual $\alpha$-level Decreasing ( $\mathrm{G} \alpha \mathrm{D}$ ) Algorithm}

Optimization on growing hyperrectangular search space from $\alpha$-level 1 to $\alpha$-level 0 .

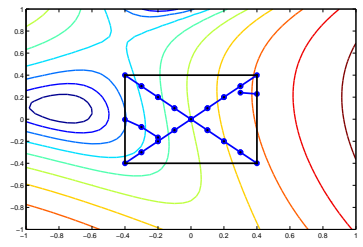

$$
\begin{aligned}
y= & \log \left(x_{1}^{3}+3.2 x_{1}^{2}+3.08 x_{1}+x_{1} x_{2}\right. \\
& \left.+0.4 x_{2}-3 x_{1} x_{2}^{2}-0.2 x_{2}^{2}-x_{2}^{3}+1\right) \\
\widetilde{x}_{1}= & \operatorname{triag}(-1,0,1) \\
\widetilde{x}_{2}= & \operatorname{triag}(-1,0,1)
\end{aligned}
$$

$$
\alpha=0.6
$$




\section{Optimization with $\mathrm{G} \alpha \mathrm{D}$}

A dedicated global optimization algorithm:

\section{Gradual $\alpha$-level Decreasing ( $\mathrm{G} \alpha \mathrm{D}$ ) Algorithm}

Optimization on growing hyperrectangular search space from $\alpha$-level 1 to $\alpha$-level 0 .

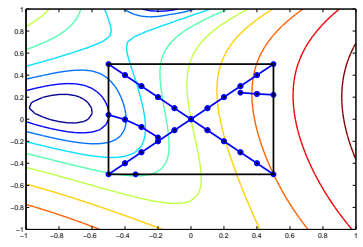

$$
\begin{aligned}
y= & \log \left(x_{1}^{3}+3.2 x_{1}^{2}+3.08 x_{1}+x_{1} x_{2}\right. \\
& \left.+0.4 x_{2}-3 x_{1} x_{2}^{2}-0.2 x_{2}^{2}-x_{2}^{3}+1\right) \\
\widetilde{x}_{1}= & \operatorname{triag}(-1,0,1) \\
\widetilde{x}_{2}= & \operatorname{triag}(-1,0,1)
\end{aligned}
$$

$$
\alpha=0.5
$$




\section{Optimization with $\mathrm{G} \alpha \mathrm{D}$}

A dedicated global optimization algorithm:

\section{Gradual $\alpha$-level Decreasing ( $\mathrm{G} \alpha \mathrm{D}$ ) Algorithm}

Optimization on growing hyperrectangular search space from $\alpha$-level 1 to $\alpha$-level 0 .

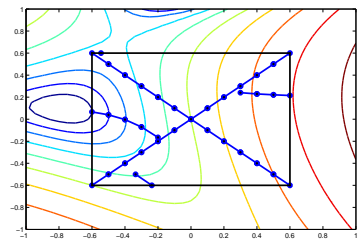

$$
\begin{aligned}
y= & \log \left(x_{1}^{3}+3.2 x_{1}^{2}+3.08 x_{1}+x_{1} x_{2}\right. \\
& \left.+0.4 x_{2}-3 x_{1} x_{2}^{2}-0.2 x_{2}^{2}-x_{2}^{3}+1\right) \\
\widetilde{x}_{1}= & \operatorname{triag}(-1,0,1) \\
\widetilde{x}_{2}= & \operatorname{triag}(-1,0,1)
\end{aligned}
$$

$$
\alpha=0.4
$$




\section{Optimization with $\mathrm{G} \alpha \mathrm{D}$}

A dedicated global optimization algorithm:

\section{Gradual $\alpha$-level Decreasing ( $\mathrm{G} \alpha \mathrm{D}$ ) Algorithm}

Optimization on growing hyperrectangular search space from $\alpha$-level 1 to $\alpha$-level 0 .

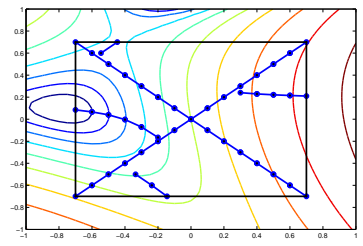

$$
\begin{aligned}
y= & \log \left(x_{1}^{3}+3.2 x_{1}^{2}+3.08 x_{1}+x_{1} x_{2}\right. \\
& \left.+0.4 x_{2}-3 x_{1} x_{2}^{2}-0.2 x_{2}^{2}-x_{2}^{3}+1\right) \\
\widetilde{x}_{1}= & \operatorname{triag}(-1,0,1) \\
\widetilde{x}_{2}= & \operatorname{triag}(-1,0,1)
\end{aligned}
$$

$$
\alpha=0.3
$$




\section{Optimization with $\mathrm{G} \alpha \mathrm{D}$}

A dedicated global optimization algorithm:

\section{Gradual $\alpha$-level Decreasing ( $\mathrm{G} \alpha \mathrm{D}$ ) Algorithm}

Optimization on growing hyperrectangular search space from $\alpha$-level 1 to $\alpha$-level 0 .

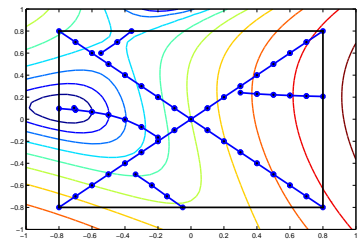

$$
\begin{aligned}
y= & \log \left(x_{1}^{3}+3.2 x_{1}^{2}+3.08 x_{1}+x_{1} x_{2}\right. \\
& \left.+0.4 x_{2}-3 x_{1} x_{2}^{2}-0.2 x_{2}^{2}-x_{2}^{3}+1\right) \\
\widetilde{x}_{1}= & \operatorname{triag}(-1,0,1) \\
\widetilde{x}_{2}= & \operatorname{triag}(-1,0,1)
\end{aligned}
$$

$$
\alpha=0.2
$$




\section{Optimization with $\mathrm{G} \alpha \mathrm{D}$}

A dedicated global optimization algorithm:

\section{Gradual $\alpha$-level Decreasing (G $\alpha \mathrm{D}$ ) Algorithm}

Optimization on growing hyperrectangular search space from $\alpha$-level 1 to $\alpha$-level 0 .

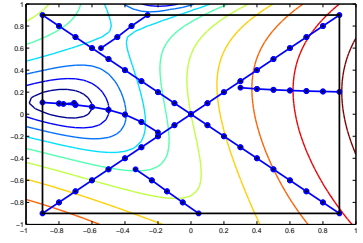

$$
\begin{aligned}
y= & \log \left(x_{1}^{3}+3.2 x_{1}^{2}+3.08 x_{1}+x_{1} x_{2}\right. \\
& \left.+0.4 x_{2}-3 x_{1} x_{2}^{2}-0.2 x_{2}^{2}-x_{2}^{3}+1\right) \\
\widetilde{x}_{1}= & \operatorname{triag}(-1,0,1) \\
\widetilde{x}_{2}= & \operatorname{triag}(-1,0,1)
\end{aligned}
$$

$$
\alpha=0.1
$$




\section{Optimization with $\mathrm{G} \alpha \mathrm{D}$}

A dedicated global optimization algorithm:

\section{Gradual $\alpha$-level Decreasing ( $\mathrm{G} \alpha \mathrm{D}$ ) Algorithm}

Optimization on growing hyperrectangular search space from $\alpha$-level 1 to $\alpha$-level 0 .

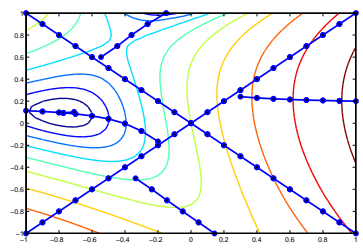

$$
\begin{aligned}
y= & \log \left(x_{1}^{3}+3.2 x_{1}^{2}+3.08 x_{1}+x_{1} x_{2}\right. \\
& \left.+0.4 x_{2}-3 x_{1} x_{2}^{2}-0.2 x_{2}^{2}-x_{2}^{3}+1\right) \\
\widetilde{x}_{1}= & \operatorname{triag}(-1,0,1) \\
\widetilde{x}_{2}= & \operatorname{triag}(-1,0,1)
\end{aligned}
$$

$$
\alpha=0
$$




\section{PDE with fuzzy parameters}

PDE of elliptic type:

$$
-\nabla \cdot(k \nabla u)+c u=f \text { in } \Omega \subset \mathbb{R}^{2} \text { s.t. } \alpha u+\beta \frac{\partial u}{\partial \vec{n}}=\gamma \text { on } \partial \Omega,
$$

Discretization results in a linear system:

$$
A(\mathbf{k}) \mathbf{u}=\mathbf{b}
$$

If we assume $k$ is a fuzzy field $\widetilde{k}$, We can fuzzify the system:

$$
A(\widetilde{\mathbf{k}}) \widetilde{\mathbf{u}}=\mathbf{b}
$$




\section{Problem Formulation}

\section{Optimization Problem}

$$
\begin{array}{r}
\forall i \in \mathcal{J}, \forall \alpha_{j} \in \mathcal{A}: \max _{\mathbf{k}} u_{i} \text { and } \min _{\mathbf{k}} u_{i} \\
\text { s.t. } A(\mathbf{k}) \mathbf{u}=\mathbf{b} \text { and } \mathbf{k} \in[\widetilde{\mathbf{k}}]_{\alpha_{j}}
\end{array}
$$

The many function and gradient evaluations in optimization differ from each other through a possibly small change in parameters. Therefore we consider component reuse to accelerate re-analysis. 


\section{Reuse in matrix assembly phase}

Assume the fuzzy field $\widetilde{\mathbf{k}}$ can be written as finite sum of basis functions: $\widetilde{\mathbf{k}}=\sum_{l=1}^{n} \widetilde{k_{l}} \varphi_{l}$, then:

$$
A(\widetilde{\mathbf{k}})=\sum_{l=1}^{n} \widetilde{k}_{l} K_{l}+M
$$

Construction of a new $A(\mathbf{k})$ in the optimization algorithm becomes very cheap once the $K_{l}$ and $M$ are known. 


\section{Reuse of earlier solution as initial guess}

Assume $A(\mathbf{k}) \mathbf{u}=\mathbf{b}$ has been solved iteratively in some iteration point $\mathbf{k}^{(i)}$ until relative residual error is below some treshold:

$$
\frac{\left\|\mathbf{b}-A^{(i)} \mathbf{u}^{(i)}\right\|}{\|\mathbf{b}\|} \leqslant \epsilon_{\text {tol }}
$$

then we can prove:

$$
\frac{\left\|\mathbf{b}-A^{(i+1)} \mathbf{u}^{(i)}\right\|}{\|\mathbf{b}\|} \leqslant \epsilon_{\mathrm{tol}}+\sum_{l=1}^{n}\left|\Delta k_{l}^{(i+1)}\right|\left\|K_{l}\right\|\left\|\left(A^{(i)}\right)^{-1}\right\|\left(1+\epsilon_{\mathrm{tol}}\right)
$$

The smaller the step, the better the earlier solution is as initial guess. 


\section{Reuse intergrid operators in algebraic multigrid}

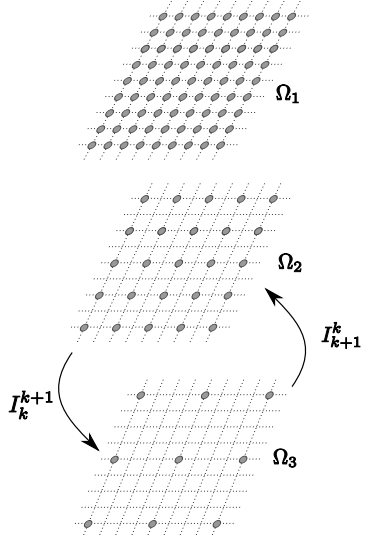

\section{Basic two-grid to solve $A \mathbf{u}=\mathbf{b}$}

(1) Choose initial guess $\mathbf{u}_{\text {init }}$

(2) relax $\nu_{1}$ times on $A_{1} \mathbf{u}_{1}=\mathbf{b}_{1}$

(3) $\mathbf{r}_{2} \leftarrow l_{1}^{2}\left(\mathbf{b}_{1}-A_{1} \mathbf{u}_{1}\right)$

(3) solve $A_{2} \mathbf{e}_{2}=\mathbf{r}_{2}$

(3) $\mathbf{u}_{1} \leftarrow \mathbf{u}_{1}+I_{2}^{1} \mathbf{e}_{2}$

(2) relax $\nu_{2}$ times on $A_{1} \mathbf{u}_{1}=\mathbf{b}_{1}$ 


\section{Reuse intergrid operators in algebraic multigrid}

- Discrete operator $A_{k}$ for grid $\Omega_{k}$ is constructed like: $A_{k+1}=I_{k}^{k+1} A_{k} l_{k+1}^{k}$, with $A_{1}=A$.

- The intergrid transfer operators $I_{k}^{k+1}$ and $I_{k+1}^{k}$ are constructed during costly setup phase in algebraic multigrid.

Therefore we construct $I_{k}^{k+1}\left(\mathbf{k}_{\text {ref }}\right)$ and $l_{k+1}^{k}\left(\mathbf{k}_{\text {ref }}\right)$ in some reference point $\mathbf{k}_{\text {ref }}$ and reuse them in other iteration points to construct $A_{k+1}=l_{k}^{k+1}\left(\mathbf{k}_{\mathrm{ref}}\right) A_{k} l_{k+1}^{k}\left(\mathbf{k}_{\mathrm{ref}}\right)$, with $A_{1}=A\left(\mathbf{k}^{(i)}\right)$. 


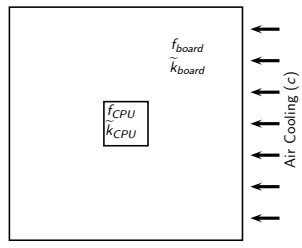

- 2 uncertain parameters: $\widetilde{\mathbf{k}}_{\mathrm{CPU}}$ and $\widetilde{\mathbf{k}}_{\text {board }}$

- grid of $100 \times 100$

- about 1000 function evaluations

- AMG: Ruge-Stüben

\begin{tabular}{ccccc}
\hline $\begin{array}{c}\text { intergrid } \\
\text { operator reuse }\end{array}$ & $\begin{array}{c}\text { initial } \\
\text { guess reuse }\end{array}$ & $\begin{array}{c}\text { multigrid } \\
\text { setup time }(\mathbf{s})\end{array}$ & $\begin{array}{c}\text { solution } \\
\text { time }(\mathbf{s})\end{array}$ & $\begin{array}{c}\text { total } \\
\text { time }(\mathbf{s})\end{array}$ \\
\hline $\mathrm{x}$ & $\mathrm{x}$ & 2.89 & 23.25 & 26.14 \\
\hline $\mathrm{x}$ & & 2.89 & 51.43 & 54.32 \\
\hline $\mathrm{x}$ & 86.24 & 22.27 & 108.51 \\
\hline & 86.71 & 49.05 & 135.76 \\
\hline
\end{tabular}

(Add about $30 \mathrm{~min}$. extra in case of no matrix assembly reuse.) 


\section{Thank You!}

